\title{
Análise genética de características reprodutivas na raça Nelore(1)
}

\author{
Evandro Pereira ${ }^{(2)}$, Joanir Pereira Eler ${ }^{(3)}$ e José Bento Sterman Ferraz ${ }^{(3)}$
}

Resumo - O objetivo deste trabalho foi estimar os coeficientes de herdabilidade da idade ao primeiro parto, dias para o parto (DPP), duração da gestação (DG) e perímetro escrotal (PE) bem como a correlação genética entre perímetro escrotal e estas características reprodutivas medidas nas fêmeas, na raça Nelore. A idade ao primeiro parto foi avaliada em fêmeas expostas pela primeira vez ao touro em torno de 14 meses de idade (IPP14), e em fêmeas expostas ao touro em torno de 26 meses (IPP26). Foram analisados conjuntos de dados que variaram de 6.030 a 94.637 observações. As análises foram processadas utilizando-se modelos animais bi-característica. Os coeficientes de herdabilidade obtidos em relação às características reprodutivas foram: 0,19 (IPP14), 0,02 (IPP26), 0,07 (DPP), 0,26 (DG), e 0,47 (PE). As correlações genéticas obtidas foram: -0,39 (PE e IPP14), -0,19 (PE e IPP26), 0,02 (PE e DPP) e 0,02 (PE e DG). Concluiu-se que a idade ao primeiro parto pode ser utilizada como critério de seleção para precocidade sexual, e que a seleção de touros com base no mérito genético para PE pode resultar na diminuição da idade ao primeiro parto de suas filhas. A baixa herdabilidade obtida quanto aos DPP, associada com a sua baixa correlação com PE, sugerem a necessidade do estudo de outras características que possam avaliar diretamente a fertilidade da fêmea.

Termos para indexação: bovinos, correlação genética, parâmetros genéticos, herdabilidade.

\section{Genetic analysis of reproductive traits in Nelore cattle}

Abstract - The objective of this paper was to estimate heritability coefficients for age at first calving, days to calving (DC), gestation length (GL) and scrotal circumference (SC) as well as genetic correlations between scrotal circumference and these reproductive traits measured in the females, in Nelore cattle. The age at first calving was evaluated in females mated for the first time around 14 months of age (AFC14) and in females mated for the first time around 26 months of age (AFC26). Datasets varying from 6,030 to 94,637 observations were analyzed. Analyses were processed using two-trait animal models. Heritability coefficients obtained for reproductive traits were: 0.19 (AFC14), 0.02 (AFC26), 0.07 (DC), 0.26 (GL), and 0.47 (SC). The genetic correlations were: - 0.39 (SC and AFC14), -0.19 (SC and $\mathrm{AFC} 26), 0.02$ (SC and DC); and 0.02 (SC and GL). It was concluded that age at first calving may be used as a selection criterion for sexual precocity and that the selection of bulls for SC can result in daughters with decreased age at first calving. The low heritability obtained for DC, associated to its low genetic correlation with SC, suggest the need of studies on other traits to evaluate female fertility.

Index terms: bovines, genetic correlation, genetic parameters, heritability.

(1) Aceito para publicação em 7 de agosto de 2001. Pesquisa apoiada pela Fapesp, pelo CNPq e pela Agropecuária CFM Ltda.

(2) Universidade de São Paulo (USP), Faculdade de Zootecnia e Engenharia de Alimentos (FZEA), Dep. de Ciências Bá sicas, Caixa Postal 23, Rua Duque de Caxias Norte, 225, CEP 13635-900 Pirassununga, SP. Bolsista da Fapesp. E-mail: epereira@1ancernet.com.br

(3) USP, FZEA, Dep. de Ciências Básicas, Caixa Postal 23, Rua Duque de Caxias Norte, 225, CEP 13635-900 Pirassununga, SP. Bolsista do CNPq E-mail: joapeler@usp.br,jbferraz@usp.br

\section{Introdução}

O perímetro escrotal está associado à qualidade do sêmen e à libido em touros jovens (até a idade de um ano para Bos taurus e dois anos para Bos indicus) e tem sido uma característica importante para a seleção de animais que possuem maior potencial para produção quantitativa e qualitativa de sêmen e, conseqüentemente, maior fertilidade (Knights et al., 1984; Moser et al., 1996; Sarreiro et al., 2000). 
A medida do perímetro escrotal também está correlacionada com a idade à puberdade em machos e fêmeas (Martin et al., 1992; Bergmann et al., 1996; Moser et al., 1996). Vários são os trabalhos que citam estimativas de correlação genética negativas entre perímetro escrotal e características reprodutivas das fêmeas (Toelle \& Robison, 1985; Martins Filho \& Lôbo, 1991; Meyer et al., 1991; Martin et al., 1992; Morris et al., 1992; Morris \& Cullen, 1994; Gressler et al., 1998; Pereira et al., 1998; Dias et al., 2000; Pereira et al., 2000a). A correlação negativa, neste caso, implica associação favorável entre as características.

A herdabilidade relativamente alta (Koots et al., 1994; Bergmann et al., 1996; Quirino \& Bergmann, 1998; Lôbo et al., 2000), a facilidade de medição e as correlações genéticas positivas com características de sêmen e negativas com características reprodutivas das fêmeas, fazem do perímetro escrotal o critério de seleção mais utilizado para melhoria da eficiência reprodutiva em gado de corte. Entretanto, nos últimos anos, tem-se questionado a eficácia de sua utilização para melhorar a precocidade e a fertilidade das fêmeas, com o argumento de que as correlações genéticas têm baixa magnitude (Pereira et al., 1998; Pereira et al., 2000b). Diante disso, haveria a necessidade de se estudar outras características medidas diretamente nas fêmeas e que pudessem ser indicadoras de precocidade e fertilidade. A idade ao primeiro parto é uma característica que vem sendo utilizada em pesquisas como indicadora da precocidade sexual (Toelle \& Robison, 1985; Martins Filho \& Lôbo, 1991; Pereira et al., 1998; Dias et al., 2000; Pereira et al., 2000a). O número de dias para o parto pode ser uma característica indicadora de fertilidade em gado de corte, segundo Johnston \& Bunter (1996) que a utiliza no BREEDPLAN (National Beef Recording Scheme of Australia). A duração da gestação, embora não seja propriamente uma medida de fertilidade, é estreitamente relacionada com o período produtivo, influenciando o número de dias para o parto e até mesmo a idade ao primeiro parto. Além disso, bezerros com gestação mais curta nascem mais leves (Lôbo et al., 2000) e tendem a desmamarem mais pesados por terem em média um maior período de tempo antes de desmamarem (considerando a desmama em um período fixo). Fêmeas com período de gestação mais curto têm maior probabilidade de sucesso na estação de monta subseqüente, pois elas parem antes e têm mais tempo de repouso antes de entrarem na nova estação de monta.

O objetivo deste trabalho foi estimar os coeficientes de herdabilidade da idade ao primeiro parto, dias para o parto, duração da gestação e perímetro escrotal, bem como a correlação genética entre o perímetro escrotal e estas características reprodutivas medidas nas fêmeas.

\section{Material e Métodos}

Os dados utilizados foram obtidos em 15 fazendas situadas nos Estados de São Paulo, Mato Grosso do Sul e Goiás, todas pertencentes ao programa de melhoramento genético da Empresa Agropecuária CFM Ltda.

Os arquivos analisados continham registros de características reprodutivas de animais da raça Nelore, nascidos entre 1971 e 1998, e criados em regime exclusivo de pasto. Eram realizadas duas estações de monta: a principal, outubro a dezembro, e a fora de época, de março a maio. Fazia parte do manejo utilizado na empresa o descarte das vacas vazias no final de cada estação de monta

As características analisadas foram: idade ao primeiro parto (IPP14 e IPP26), dias para o parto (DPP), duração da gestação (DG) e perímetro escrotal (PE) medido em torno de 18 meses (14 a 22 meses)

A idade ao primeiro parto, em dias, foi obtida pela diferença entre a data do primeiro parto e a data de nascimento da fêmea. $\mathrm{O}$ arquivo total de idade ao primeiro parto foi dividido em dois, de acordo com a idade à primeira exposição ao touro: novilhas expostas ao touro pela primeira vez em torno de 14 meses (12 a 16 meses); e novilhas expostas ao touro pela primeira vez em torno de 26 meses (22 a 30 meses). Portanto, foram analisadas duas características relacionadas com a idade ao primeiro parto: IPP14 e IPP26.

No cálculo de DG, foram utilizados somente registros de fêmeas inseminadas artificialmente. A DG foi analisada como característica da vaca e, portanto, com registros repetidos, por animal.

A variável DPP também foi considerada como medida repetida e corresponde ao número de dias entre o primeiro dia da estação de monta e o parto, para cada estação de monta. Foi ajustada para sexo do bezerro, subtraindo-se dois dias para as vacas que pariram machos, pois a DG de vacas que pariram machos foi, em média, dois dias superior à $\mathrm{DG}$ das vacas que pariram fêmeas.

As medidas de PE foram feitas transversalmente, na região de maior diâmetro do escroto, com a utilização de fita métrica metálica. 
Foram processadas, inicialmente, análises utilizando-se o pacote estatístico SAS Institute (1990), procedimento GLM. Objetivou-se estudar os fatores não-genéticos que estariam influenciando as características em questão, e identificar aqueles com efeitos significativos. A partir deste estudo inicial, foram definidos os grupos contemporâneos e as covariáveis incluídas nos modelos para as análises genéticas. Grupos contemporâneos com apenas uma observação foram eliminados.

$\mathrm{Na}$ análise genética, o método empregado foi o de modelos mistos, utilizando modelo animal (Henderson, 1975)

O modelo animal bi-característica utilizado foi uma forma especial, no qual cada característica era medida em apenas um sexo. Assim, as características foram conectadas apenas pela matriz de parentesco. Para este modelo, a covariância residual entre as características foi assumida como zero.

Os efeitos fixos utilizados para cada característica foram:

IPP14 e IPP26: grupo contemporâneo, que incluiu ano de nascimento + estação de nascimento (1: janeiro a agosto; 2: setembro a dezembro) + fazenda de nascimento + grupo de manejo à desmama + grupo de manejo ao sobreano + fazenda da reprodução + ano da primeira exposição + estação de monta da primeira exposição (1: março a maio; 2: outubro a dezembro) e classe de idade da mãe ao parto ( 7 classes), além da idade à primeira exposição ao touro, como covariável linear;

DPP: grupo contemporâneo, que incluiu fazenda da reprodução + ano da reprodução + estação de monta (1: março a maio; 2: outubro a dezembro) + pai do bezerro (ou grupo de reprodutores múltiplos, $\mathrm{RM}$ ) + categoria de idade no início da estação de monta (4 classes);

DG: grupo contemporâneo, que consistiu de ano de nascimento + estação de nascimento ( 1 ou 2 , como definido anteriormente) + fazenda da reprodução + ano da reprodução + estação de monta ( 1 ou 2 , como definido anteriormente), além de sexo do produto (1: macho; 2 : fêmea), raça do produto (1: Nelore puro; 2 : cruzado) e classe de idade da vaca ao parto (6 classes);

PE: grupo contemporâneo, consistindo de fazenda de nascimento + ano de nascimento + estação de nascimento ( 1 ou 2, como definido anteriormente) + grupo de manejo ao sobreano, além de idade à medição como covariável linear.

Os componentes de (co)variância foram estimados por máxima verossimilhança restrita, utilizando-se o software MTDFREML - Multiple Trait Derivative Free Restricted Maximum Likelihood (Boldman et al., 1993).

\section{Resultados e Discussão}

As médias encontradas de cada característica estudada são comparáveis às médias descritas na literatura, para a raça Nelore (Tabela 1).

O coeficiente de herdabilidade para IPP14 foi superior ao obtido para IPP26 (Tabela 2). Isto pode ter ocorrido devido ao fato de as fêmeas expostas mais velhas já terem atingido a puberdade antes da primeira exposição, o que tornou mais difícil a detecção de diferenças genéticas entre elas. $\mathrm{O}$ mesmo parece não ter ocorrido quando as fêmeas foram expostas mais cedo, o que permitiu a detecção de diferenças entre as idades à puberdade. Assim, a utilização desta característica como critério de seleção para precocidade sexual seria mais recomendada para uma população de fêmeas expostas ao touro ainda jovens (em torno de 14 meses). Ambos os coeficientes de herdabilidade estão dentro da faixa de valores encontrada na literatura sobre a raça Nelore (Gressler et al., 1998; Dias et al., 2000). É interessante observar, ainda, que, embora as novilhas dos dois grupos (IPP14 e IPP26) tenham sido expostas ao touro com uma diferença média de 12 meses de idade, a diferença entre as médias de idade ao primeiro parto foi de cerca de três meses $(33$ x 36 meses) (Tabela 1). Isto porque a maior parte das novilhas expostas em torno de 14 meses não conceberam à primeira exposição, aumentando, assim, a magnitude dos componentes de variância obtidos para IPP14 (Tabela 2). As novilhas que não concebiam à primeira exposição tinham oportunidade de conceber na próxima estação, aumentando a variabilidade dos dados de idade ao primeiro parto na população.

A herdabilidade da DPP foi 0,07 , e a repetibilidade $\left(\mathrm{h}_{\mathrm{a}}{ }^{2}+\mathrm{c}^{2}\right), 0,16$ (Tabela 2). Estes resultados estão dentro da faixa de amplitude observada na literatura (Meyer et al., 1990, 1991; Johnston \& Bunter, 1996). A baixa herdabilidade desta característica indica que há pequena variância genética aditiva (Tabela 2). Entretanto, a DPP é uma característica que só foi avaliada recentemente na população objeto deste estudo. Assim, os conjuntos de dados das safras mais antigas não possuem identificação correta dos lotes de vacas na reprodução. Pode ser que esta falta de identificação tenha levado a grupos contemporâneos inadequados, aumentando a variância residual. 
Jonhston \& Bunter (1996) obtiveram, em uma das suas estimativas, coeficiente de herdabilidade igual a 0,14 , valor este utilizado pelo BREEDPLAN na predição do mérito genético (DEPs) para a característica. A utilização de herdabilidade menor do que 0,14 em programas de melhoramento teria como conseqüência, pequena resposta à seleção e exigiria que os reprodutores tivessem grande número de filhas avaliadas para que as DEPs apresentassem confiabilidade (acurácia) suficiente.

Com relação à DG, o valor de herdabilidade encontrado $(0,26)$ (Tabela 2$)$ é superior à média relatada por Lôbo et al. (2000). Este valor de herdabilidade permite boa resposta à seleção para esta característica. A contribuição do pai do bezerro para a variância fenotípica foi de $17 \%$ (Tabela 2), o que mostra a importância de se considerar este efeito aleatório no modelo para DG. Esta característica não tem sido utilizada como critério de seleção, embora ela possa ser uma ferramenta importante no monitoramento do peso ao nascer, que vem aumentando bastante nos programas de melhoramento devido à correlação elevada com peso à desmama. Ferraz \& Eler (2000) mostram uma tendência genética igual a $0,2 \mathrm{~kg}$ para o peso ao nascer na raça Nelore.

O valor do coeficiente de herdabilidade encontrado para PE $(0,47)$ (Tabela 2$)$ está dentro da faixa de valores encontrada na literatura em relação à raça Nelore, que varia de 0,31 (Gressler et al., 1998) a 0,77 (Quirino \& Bergmann, 1998). A seleção para perímetro escrotal poderia levar a ganhos genéticos elevados para esta característica.

As correlações de IPP14 e IPP26 com PE foram negativas, $-0,39$ e $-0,19$, respectivamente (Tabela 2 ). Isto indica que o $\mathrm{PE}$ pode ser utilizado como critério de seleção para melhorar a precocidade sexual das fêmeas, uma vez que a correlação genética negativa indica que quanto maior o PE de um touro, menor a IPP14 e a IPP26 de suas filhas. Pode-se observar, ainda, que a magnitude da correlação foi maior em relação à IPP14. Como mencionado anteriormente, esta característica deve refletir melhor as diferenças entre as idades à puberdade das fêmeas do que IPP26. Valores negativos para esta correlação genética são

Tabela 1. Número de observações $(\mathrm{N})$, média ( $\overline{\mathrm{x}}$ ), desvio-padrão (DP), coeficiente de variação $(\mathrm{CV})$, valores mínimo (MIN) e máximo (MAX) e número de grupos contemporâneos (NGC) para características reprodutivas.

\begin{tabular}{|c|c|c|c|c|c|c|c|}
\hline Característica $^{(1)}$ & $\mathrm{N}$ & $\overline{\mathrm{x}}$ & $\mathrm{DP}$ & $\mathrm{CV}$ & MIN & MAX & NGC \\
\hline IPP14 (dias) & 6.030 & $1.006,61$ & 132,19 & 13,13 & 637 & 1.520 & 278 \\
\hline IPP26 (dias) & 49.268 & $1.099,80$ & 49,77 & 4,53 & 894 & 1.574 & 2.554 \\
\hline DPP (dias) & 94.637 & 320,38 & 21,54 & 6,72 & 268 & 400 & 1.806 \\
\hline DG (dias) & 31.016 & 290,60 & 11,35 & 3,91 & 235 & 310 & 882 \\
\hline $\mathrm{PE}(\mathrm{cm})$ & 25.358 & 27,75 & 3,25 & 11,70 & 15 & 44 & 974 \\
\hline
\end{tabular}

(1)IPP14 e IPP26: idade ao primeiro parto para fêmeas expostas ao touro pela primeira vez em torno de 14 e 26 meses, respectivamente; DPP: dias para o parto; DG: duração da gestação; PE: perímetro escrotal.

Tabela 2. Parâmetros genéticos para idade ao primeiro parto (IPP14 e IPP26), dias para o parto (DPP), duração da gestação (DG) e perímetro escrotal (PE) em análises bi-característica.

\begin{tabular}{|c|c|c|c|c|c|c|c|c|c|}
\hline \multirow[t]{2}{*}{ Análise } & \multicolumn{9}{|c|}{ Parâmetros genéticos ${ }^{(1)}$} \\
\hline & $\sigma_{\mathrm{a}}{ }^{2}$ & $\sigma_{c}^{2}$ & $\sigma_{t}^{2}$ & $\sigma_{\mathrm{e}}{ }^{2}$ & $\mathrm{~h}_{\mathrm{a}}{ }^{2}$ & $\mathrm{c}^{2}$ & $\mathrm{t}^{2}$ & $\mathrm{e}^{2}$ & $\mathrm{rg}$ \\
\hline $\mathrm{IPP} 14+\mathrm{PE}$ & $2.401,71$ & - & - & $10.142,32$ & 0,19 & - & - & 0,81 & $-0,39$ \\
\hline IPP26 + PE & 25,80 & - & - & $1.500,62$ & 0,02 & - & - & 0,98 & $-0,19$ \\
\hline $\mathrm{DPP}+\mathrm{PE}$ & 21,49 & 31,03 & - & 282,72 & 0,07 & 0,09 & - & 0,84 & 0,02 \\
\hline $\mathrm{DG}+\mathrm{PE}$ & 17,96 & - & 11,76 & 39,10 & 0,26 & - & 0,17 & 0,57 & 0,02 \\
\hline PE + IPP14 & 3,00 & - & - & 3,41 & 0,47 & - & - & 0,53 & - \\
\hline$P E+I P P 26$ & 2,99 & - & - & 3,42 & 0,47 & - & - & 0,53 & - \\
\hline $\mathrm{PE}+\mathrm{DPP}$ & 2,99 & - & - & 3,42 & 0,47 & - & - & 0,53 & - \\
\hline $\mathrm{PE}+\mathrm{DG}$ & 3,00 & - & - & 3,42 & 0,47 & - & - & 0,53 & - \\
\hline
\end{tabular}

${ }^{(1)} \sigma^{2}$ : variância genética aditiva direta; $\sigma_{c}^{2}$ : variância devida ao efeito aleatório do ambiente permanente da vaca; $\sigma_{t}^{2}$ : variância devida ao efeito aleatório do pai do bezerro; $\sigma_{\mathrm{e}}^{2}$ : variância ambiental; $\mathrm{h}_{\mathrm{a}}{ }^{2}$ : estimativa do coeficiente de herdabilidade para efeitos genéticos diretos; $\mathrm{c}^{2}$ : fração da variância fenotípica devida ao ambiente permanente da vaca; $\mathrm{t}^{2}$ : fração da variância fenotípica devida ao pai do bezerro; $\mathrm{e}^{2}$ : fração da variância fenotípica devida aos efeitos residuais; rg: correlação genética entre a característica da fêmea e PE. 
descritos na literatura mundial (Toelle \& Robison, 1985; Martins Filho \& Lôbo, 1991; Martin et al., 1992; Gressler et al., 1998; Pereira et al., 1998; Dias et al., 2000; Pereira et al., 2000a).

A correlação muito baixa entre PE e DPP $(0,02)$ indica que a seleção em relação ao PE praticamente não tem influência na fertilidade do rebanho (avaliada por DPP). A literatura mostra resultados controversos quanto à correlação genética entre PE e DPP ou PE e data do parto (característica equivalente a DPP), apresentando valores positivos (Morris \& Cullen, 1994; Gressler et al., 1998), em concordância com o aqui encontrado, e negativos (Meyer et al., 1991; Pereira et al., 1998). A baixa herdabilidade de $\mathrm{DPP}$, associada à baixa correlação genética entre $\mathrm{PE}$ e DPP, sugere a necessidade do estudo de outras características que possam avaliar diretamente a fertilidade da fêmea e que tenham maior herdabilidade. Uma destas características é a habilidade de permanência das vacas no rebanho ou "stayability" (Snelling et al., 1995). Estes autores encontraram valores de herdabilidade para a habilidade de permanência variando de 0,11 a 0,14 . Esta característica foi definida como a probabilidade da fêmea ter cinco bezerros, dado que ela pariu uma vez.

A correlação entre PE e DG também foi muito baixa $(0,02)$, de forma que a seleção para maior PE praticamente não exerceria efeito na DG.

\section{Conclusões}

1. A idade ao primeiro parto pode ser utilizada como critério de seleção para precocidade sexual, principalmente para a seleção de touros, nos quais a confiabilidade da predição do mérito genético é maior.

2. A utilização da idade ao primeiro parto como critério de seleção pode apresentar maior resposta em populações nas quais as fêmeas entram na estação de monta em torno dos 14 meses de idade.

3. A baixa herdabilidade obtida para a característica dias para o parto contra-indica sua utilização como critério de seleção na raça Nelore.

4. A seleção de touros com base no mérito genético para perímetro escrotal pode resultar na diminuição da idade ao primeiro parto de suas filhas.

\section{Referências}

BERGMANN, J. A. G.; ZAMBORLINI, L. C.; PROCÓPIO, C. S. O.; ANDRADE, V. J.; VALE FILHO, V. R. Estimativas de parâmetros genéticos do perímetro escrotal e do peso corporal em animais da raça Nelore. Arquivo Brasileiro de Medicina Veterinária e Zootecnia, Belo Horizonte, v. 48, p. 69-78, 1996.

BOLDMAN, K. G.; VLECK, L. D. van; KRIESE, L. A. A manual for use of MTDFREML: a set of programs to obtain estimates of variances and covariances. [S.1.] : Usda, 1993. $114 \mathrm{p}$.

DIAS, L. T.; FARO, L. E.; FRIES, L. A.; ALBUQUERQUE, L. G. Estimativas de parâmetros genéticos para perímetro escrotal e idade ao primeiro parto em animais da raça Nelore. In: REUNIÃO ANUAL DA SOCIEDADE BRASILEIRA DE ZOOTECNIA, 37., 2000, Viçosa, MG. Anais... Viçosa, MG: Sociedade Brasileira de Zootecnia, 2000. 1 CD.

FERRAZ, J. B. S.; ELER, J. P. Sumário de touros Nelore 2000. São José do Rio Preto: Agropecuária CFM, 2000. $60 \mathrm{p}$.

GRESSLER, S. L.; BERGMANN, J. A. G.; PENNA, V. M.; PEREIRA, C. S.; PEREIRA, J. C. C. Estudo das associações genéticas entre perímetro escrotal e características reprodutivas de fêmeas da raça Nelore. In: REUNIÃO ANUAL DA SOCIEDADE BRASILEIRA DE ZOOTECNIA, 35., 1998. Botucatu. Anais... Botucatu: Sociedade Brasileira de Zootecnia, 1998. v. 3, p. 368-370.

HENDERSON, C. R. Best linear unbiased prediction under a selection model. Biometrics, Washington, v. 31, p. 423447,1975

JOHNSTON, D. J.; BUNTER, K. L. Days to calving in Angus cattle: genetic and environmental effects, and covariances with other traits. Livestock Production Science, Amsterdam, v. 45, p. 13-22, 1996.

KNIGHTS, S. A.; BAKER, R. L.; GIANOLA, D.; GIBB, J. B. Estimates of heritabilities and of genetic and phenotypic correlations among growth and reproductive traits in yearling Angus bulls. Journal of Animal Science, Savoy, v. 58, p. 887-893, 1984

KOOTS, K. R.; GIBSON, J. P.; SMITH, C.; WILTON, $\mathrm{J}$. W. Analysis of published genetic parameter estimates for beef production traits 1: heritability. Animal Breeding Abstracts, Wallingford, v. 62, p. 309-338, 1994.

LÔBO, R. N. B.; MADALENA, F. E.; VIEIRA, A. R. Average estimates of genetic parameters for beef and dairy cattle in tropical regions. Animal Breeding Abstracts, Wallingford, v. 68, p. 433-462, 2000. 
MARTIN, L. C.; BRINKS, J. S.; BOURDON, R. M.; CUNDIFF, L. V. Genetic effects on beef heifer puberty and subsequent reproduction. Journal of Animal Science, Savoy, v. 70, p. 4006-4017, 1992.

MARTINS FILHO, R.; LÔBO, R. B. Estimates of genetic correlations between sire scrotal circumference and offspring age at first calving in Nellore cattle. Revista Brasileira de Genética, Ribeirão Preto, v. 14, p. 209 212, 1991 .

MEYER, K.; HAMMOND, K.; MacKINNON, M. J.; PARNELL, P. F. Estimates of covariances between reproduction and growth in Australian beef cattle. Journal of Animal Science, Savoy, v. 69, p. 3533-3543, 1991

MEYER, K.; HAMMOND, K.; PARNELL, P. F.; MACKINNON, M. J.; SIVARAJASINGAM, S. Estimates of heritability and repeatability for reproductive traits in Australian beef cattle. Livestock Production Science, Amsterdam, v. 25, p. 15-30, 1990.

MORRIS, C. A.; BAKER, R. L.; CULLEN, N. G. Genetic correlations between pubertal traits in bulls and heifers. Livestock Production Science, Amsterdam, v. 31, p. 221234, 1992

MORRIS, C. A.; CULLEN, N. G. A note on genetic correlation between pubertal traits of males or females and lifetime pregnancy rate in beef cattle. Livestock Production Science, Amsterdam, v. 39, p. 291-297, 1994.

MOSER, D. W.; BERTRAND, J. K.; BENYSHEK, L. L.; McCANN, M. A; KISER, T. E. Effects of selection for scrotal circumference in Limousin bulls on reproductive and growth traits of progeny. Journal of Animal Science, Savoy, v. 74, p. 2052-2057, 1996.

PEREIRA, E.; ELER, J. P.; FERRAZ, J. B. S. Correlação entre perímetro escrotal e algumas características reprodutivas na raça Nelore. Revista Brasileira de Zootecnia, Viçosa, MG, v. 29, p. 1676-1683, 2000b.
PEREIRA, E.; ELER, J. P.; FERRAZ, J. B. S.; COSTA, F. A. A.; MENDONÇA, C. D. A. Análise genética da correlação entre idade ao primeiro parto e perímetro escrotal em um rebanho Nelore. In: REUNIÃO ANUAL DA SOCIEDADE BRASILEIRA DE ZOOTECNIA, 37., 2000 , Viçosa, MG. Anais... Viçosa, MG: Sociedade Brasileira de Zootecnia, 2000a. 1 CD

PEREIRA, E.; ELER, J. P.; FERRAZ, J. B. S.; FIGUEIREDO, L. G. G. Correlação entre perímetro escrotal e algumas características reprodutivas na raça Nelore. In: CONGRESSO BRASILEIRO DAS RAÇAS ZEBUÍNAS, 3., 1998, Uberaba. Anais... Uberaba: Associação Brasileira dos Criadores de Zebu, 1998. p. 381384

QUIRINO, C. R.; BERGMANN, J. A. G. Heritability of scrotal circumference adjusted and unadjusted for body weight in Nellore bulls, using univariate and bivariate animal models. Theriogenology, New York, v. 49, p. 13891396, 1998.

SARREIRO, L. C.; QUIRINO, C. R.; PINEDA, N. R.; BERGMANN, J. A. G. Associações genéticas entre libido, perímetro escrotal e qualidade de sêmen de tourinhos da raça Nelore. In: SIMPÓSIO NACIONAL DE MELHORAMENTO ANIMAL, 3., 2000, Belo Horizonte. Anais... Belo Horizonte: Sociedade Brasileira de Melhoramento Animal, 2000. p. 449-451

SAS INSTITUTE (Cary, Estados Unidos). User's guide statistics. Cary, 1990. $846 \mathrm{p}$

SNELLING, W. M.; GOLDEN, B. L.; BOURDON, R. M Within-herd genetic analysis of stayability of beef females. Journal of Animal Science, Savoy, v. 73, p. 993-1001, 1995.

TOELLE, V. D.; ROBISON, O. W. Estimates of genetic correlations between testicular measurements and female reproductive traits in cattle. Journal of Animal Science, Savoy, v. 60, p. 89-100, 1985 